Commun. Fac. Sci. Univ. Ank. Ser. A1 Math. Stat.

Volume 68, Number 2, Pages 1482-1491(2019)

DOI: $10.31801 /$ cfsuasmas. 540631

ISSN 1303-5991 E-ISSN 2618-6470

http://communications.science.ankara.edu.tr/index.php?series=A1

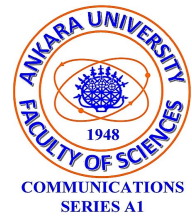

\title{
A NUMERICAL SOLUTION STUDY ON SINGULARLY PERTURBED CONVECTION-DIFFUSION NONLOCAL BOUNDARY PROBLEM
}

\author{
DERYA ARSLAN AND MUSA CAKIR
}

\begin{abstract}
This important numerical method is given for the numerical solution of singularly perturbed convection-diffusion nonlocal boundary value problem. First, the behavior of the exact solution is analyzed, which is needed for analysis of the numerical solution in later sections. Next, uniformly convergent finite difference scheme on a Shishkin mesh is established, which is based on the method of integral identities with the use exponential basis functions and interpolating quadrature rules with weight and remainder term in integral form. It is shown that the method is first order accurate expect for a logarithmic factor, in the discrete maximum norm. Finally, the numerical results are presented in table and graphs, and these results reveal the validity of the theoretical results of our method.
\end{abstract}

\section{INTRODUCTION}

In this work, we consider singularly perturbed convection-diffusion problem with nonlocal boundary value

$$
\begin{gathered}
-\varepsilon u^{\prime \prime}(x)+a(x) u^{\prime}(x)+b(x) u(x)=f(x), \quad 0<x<1, \\
u(0)=A, \\
u(1)-\sum_{i=1}^{m-2} c_{i} u\left(s_{i}\right)=B,
\end{gathered}
$$

where $0<\varepsilon<<1$ is a small perturbation parameter, $B$ and $c_{i}$ are given constants, $0<s_{1}<s_{2}<\ldots<s_{m-2}<1, i=1,2, \ldots m-2 ;$ and $a(x) \geq \alpha>0 ;$ and $a(x)$, $b(x)$ and $f(x)$ are assumed to be sufficiently continuously differentiable functions in $[0,1]$.

Received by the editors: February 05, 2018; Accepted: July 25, 2018.

2010 Mathematics Subject Classification. 65L10,65L11, 65L12, 65L15, 65L20, 65L70, 34B10.

Key words and phrases. Singular perturbation, finite difference scheme, Shishkin mesh, uniformly convergence, nonlocal condition.

Submitted via International Conference on Current Scenario in Pure and Applied Mathematics [ICCSPAM 2018].

(C)2019 Ankara University Communications Faculty of Sciences University of Ankara-Series A1 Mathematics and Statistics 
It is a well known fact that differential equations with a small parameter $\varepsilon$ multiplying the highest-order derivative terms are called singularly perturbed differential equations. Standard discretization methods for solving singular perturbation problem are unstable and these don't give accurate results for $\varepsilon$. Therefore, it is very important to find suitable numerical methods to these problems. In order to solve these problems, there are some fitted numerical approaches namely, finite difference methods, finite element methods, etc. So, we prefer to use finite difference method for solving this problem in this paper.

The first time, nonlocal boundary value problems have been studied by Bitsadze and Samarskii [5]. Singular perturbation problems arise in chemical-reactor theory, control theory, oceanography, fluid mechanics, quantum mechanics, hydro mechanical problems, meteorology, electrical networks and other physical models [13, 14, $16,17,18,19]$. Singularly perturbed differential equations with nonlocal boundary value have been studied by many authors. According to some references, existence and uniqueness of nonlocal problems can be seen in [1,4]. A finite difference scheme on an uniform mesh for solving linear (nonlinear) singularly perturbed problem with nonlocal condition have been found in $[1,2,3,6,7,8,9,10,11,12,15]$.

The plan of the study is as follows: We evaluate that for the numerical solution of the nonlocal problem (1.1)-(1.3), this method is uniformly convergent of first order on Shishkin mesh, in discrete maximum norm, independently of singular perturbation parameter $\varepsilon$. Some properties of the exact solution of problem described in (1.1)-(1.3) is investigated in Section 2. Finite difference schemes on Shishkin mesh for problem (1.1)-(1.3) are constructed in Section 3. Finite difference schemes are based on the method of integral identities with the use of exponential basis functions and interpolating quadrature rules with the weight and remainder terms in integral form [4]. The error analysis for the difference scheme is performed in Section 4. Uniform convergence is obtained in the discrete maximum norm. The iterative algorithm for solving the discrete problem is arranged and numerical example is presented to find the solution of approximation in Section 5. Throughout the paper, $C$ will mean a positive constant independent of $\varepsilon$ and the mesh parameter.

\section{Some Properties of the Continuous Problem}

In this section, we give useful asymptotic estimates of the exact solution of the problem (1.1) - (1.3), which are needed in the construction of layer-adapted meshes and examine of the numerical solution.

Lemma 2.1. Let $a(x), b(x)$ and $f(x)$ be sufficiently smooth on interval $[0,1]$ and

$$
w(1)-\sum_{i=1}^{m-2} c_{i} w\left(s_{i}\right) \neq 0,
$$

where $w(x)$ is the solution of the following problem:

$$
-\varepsilon w^{\prime \prime}+a(x) w^{\prime}(x)+b(x) w(x)=0,
$$




$$
w(0)=0, w(1)=1 .
$$

Then, the solution of problem (1.1)-(1.3) satisfies the following inequalities:

$$
\|u(x)\|_{C[0,1]} \leq C_{0}
$$

where

$$
C_{0}=|v(x)|+|\lambda||w(x)|,
$$

and

$$
\left|u^{\prime}(x)\right| \leq C_{1}\left\{1+\frac{1}{\varepsilon}\left(e^{-\frac{\alpha(1-x)}{\varepsilon}}\right)\right\}, \quad 0<x<1 .
$$

Proof. Let us take $u(1)=\lambda$ and the solution of the problem 1.1 -1.3$)$ as $u(x)=$ $v(x)+\lambda w(x)$, where

$$
\lambda=\frac{b-v(1)+\sum_{i=1}^{m-2} c_{i} v\left(s_{i}\right)}{w(1)-\sum_{i=1}^{m-2} c_{i} w\left(s_{i}\right)},
$$

and the function $v(x)$ and $w(x)$ is the solution of the following problems:

$$
\begin{gathered}
L v=f(x), \\
v(0)=A, v^{\prime}(0)=0, \\
L w=0, \\
w(0)=0, w^{\prime}(0)=1,
\end{gathered}
$$

According to the maximum principle, we have the inequalities

$$
|v(x)|=|v(0)|+\left|v^{\prime}(0)\right|+\alpha^{-1}\|f(x)\|_{C[0,1]} \leq C_{1},
$$

and

$$
|w(x)|=|w(0)|+\left|w^{\prime}(0)\right| \leq 1 .
$$

Finally, from (2.4) and (2.5), we obtain

$$
|u(x)|=|v(x)|+|\lambda||w(x)| \leq C_{1}+1 \leq C_{0},
$$

which proves $(2.2)$.

Now, we will examine the inequality (2.3). Differentiating the equation (1.1), we get the relation

$$
-\varepsilon u^{\prime \prime}(x)+a(x) u^{\prime}(x)=G(x),
$$

where

$$
f(x)-b(x) u(x)=G(x) .
$$

After doing some calculation in the equation (2.6), we have

$$
\left|u^{\prime}(x)\right| \leq C_{1}\left\{1+\frac{1}{\varepsilon}\left(e^{-\frac{\alpha(1-x)}{\varepsilon}}\right)\right\},
$$

(see in [8]). Thus, we prove (2.3). This completes the proof of Lemma 2.1. 


\section{Discretization and Mesh}

In this section, we give a difference scheme for solving our problem on Shishkin mesh.

Let us consider the following any non-uniform mesh on the interval $[0,1]$,

$$
\omega_{N}=\left\{0<x_{1}<x_{2}<\ldots<x_{N-1}<1\right\}
$$

and

$$
\bar{\omega}_{N}=\omega_{N} \cup\left\{x_{0}=0, x_{N}=1\right\} .
$$

We set the step-size $h_{i}=x_{i}-x_{i-1}, i=1,2, \ldots, N$. Before describing our numerical method, we introduce some notations for the mesh functions. We define the following finite difference for any mesh function $g_{i}=g\left(x_{i}\right)$ given on $\bar{\omega}_{N}$ :

$$
\begin{gathered}
g_{\bar{x}, i}=\frac{g_{i}-g_{i-1}}{h_{i}}, \quad g_{x, i}=\frac{g_{i+1}-g_{i}}{h_{i+1}}, \quad g_{0, i}=\frac{g_{x, i}+g_{\bar{x}, i}}{2}, \\
g_{\widehat{x}, i}=\frac{g_{i+1}-g_{i}}{\hbar_{i}}, \quad g_{\bar{x} \widehat{x}, i}=\frac{g_{x, i}-g_{\bar{x}, i}}{\hbar_{i}}, \quad \hbar_{i}=\frac{h_{i}+h_{i+1}}{2}, \\
\|g\|_{\infty} \equiv\|g\|_{\infty, \bar{\omega}_{N}}:=\max _{0 \leqslant i \leqslant N}\left|g_{i}\right| .
\end{gathered}
$$

Now, we will construct the difference scheme for the equation 1.1). First, we integrate the equation 1.1 over $\left(x_{i-1}, x_{i+1}\right)$,

$$
\hbar_{i}^{-1} \int_{x_{i-1}}^{x_{i+1}} L u(x) \varphi_{i}(x) d x=\hbar_{i}^{-1} \int_{x_{i-1}}^{x_{i+1}} f(x) \varphi_{i}(x) d x, i=\overline{1, N-1},
$$

with the basis functions $\left\{\varphi_{i}(x)\right\}_{i=1}^{N-1}$ having the from

$$
\varphi_{i}(x)=\left\{\begin{array}{cc}
\varphi_{i}^{(1)}(x)=\frac{e^{\frac{a_{i}\left(x-x_{i-1}\right)}{\varepsilon}}-1}{e^{\frac{a_{i} h_{i}}{\varepsilon}}-1}, & x_{i-1}<x<x_{i}, \\
\varphi_{i}^{(2)}(x)=\frac{1-e^{\frac{a_{i}\left(x-x_{i+1}\right)}{\varepsilon}}}{1-e^{-\frac{a_{i} h_{i+1}}{\varepsilon}}} & x \notin\left(x_{i-1}, x_{i+1}\right),
\end{array}\right.
$$

where $\varphi_{i}^{(1)}(x)$ and $\varphi_{i}^{(2)}(x)$, respectively, are the solution of the following problems:

$$
\begin{aligned}
-\varepsilon \varphi^{\prime \prime}+a_{i} \varphi^{\prime} & =0, \quad x_{i-1}<x<x_{i} \\
\varphi\left(x_{i-1}\right) & =0, \quad \varphi\left(x_{i}\right)=1 \\
-\varepsilon \varphi^{\prime \prime}+a_{i} \varphi^{\prime} & =0, \quad x_{i}<x<x_{i+1} \\
\varphi\left(x_{i}\right) & =1, \quad \varphi\left(x_{i+1}\right)=0 .
\end{aligned}
$$

After doing some arrangements in the equation (3.1), we obtain the following equation: 


$$
\varepsilon \hbar_{i}^{-1} \int_{x_{i-1}}^{x_{i+1}} u^{\prime}(x) \varphi_{i}^{\prime}(x) d x+a_{i} \hbar_{i}^{-1} \int_{x_{i-1}}^{x_{i+1}} u^{\prime}(x) \varphi(x) d x+b_{i} u_{i}=f_{i}+R_{a, i}+R_{b, i}
$$

where

$$
\begin{aligned}
R_{i}=f_{i}+R_{a, i}+R_{b, i}=\quad & \hbar_{i}^{-1} \int_{x_{i-1}}^{x_{i+1}}\left[a\left(x_{i}\right)-a(x)\right] u^{\prime}(x) \varphi_{i}(x) d x \\
& +\hbar_{i}^{-1} \int_{x_{i-1}}^{x_{i+1}}\left[b\left(x_{i}\right)-b(x)\right] u(x) \varphi_{i}(x) d x \\
& +\hbar_{i}^{-1} \int_{x_{i-1}}^{x_{i+1}}\left[f(x)-f\left(x_{i}\right)\right] \varphi_{i}(x) d x .
\end{aligned}
$$

Using the interpolating quadrature rules (2.1) and (2.2) from [4] with weight functions $\varphi_{i}(x)$ on subintervals $\left(x_{i-1}, x_{i+1}\right)$ from (3.1), we obtain the following precise relation:

$$
l u_{i}:=-\varepsilon \theta_{i} u_{\widehat{x} \widehat{x}, i}+\eta_{i} u_{\widehat{x}, i}+b_{i} u_{i}=f_{i}+R_{a, i}+R_{b, i}=R_{i}, i=\overline{1, N-1},
$$

where

$$
\theta_{i}=\frac{\frac{a_{i} h_{i}}{\varepsilon}}{1-e^{-\frac{a_{i} h_{i}}{\varepsilon}}}
$$

and

$$
\eta_{i}=\frac{-a_{i} h_{i}}{h_{i+1}\left[1-e^{-\frac{a_{i} h_{i}}{\varepsilon}}\right]}+\frac{a_{i}}{1-e^{\frac{a_{i} h_{i+1}}{\varepsilon}}}
$$

Thus, by neglecting $R_{i}$ in the equation (3.4), we suggest the following difference scheme for approximating (1.1)-(1.3):

$$
\begin{gathered}
l y_{i}:=-\varepsilon \theta_{i} y_{\bar{x} \widehat{x}, i}+\eta_{i} y_{\widehat{x}, i}+b_{i} y_{i}=f_{i}, i=\overline{1, N-1}, \\
y_{0}=A, \\
y_{N}=\sum_{i=1}^{m-2} c_{i} y_{N_{i}}\left(x_{N_{i}}\right)+B,
\end{gathered}
$$

where $x_{N_{i}}$ is the mesh point nearest to $s_{i}, \theta_{i}$ and $\eta_{i}$ are given by 3.5 and 3.6 


\section{UNiform ERror Estimate}

In this section, we obtain the convergence of the method. First, we give the error function $z_{i}=y_{i}-u_{i}, i=0,1, \ldots, N$, where $z_{i}$ is the solution of the discrete problem

$$
\begin{gathered}
-\varepsilon \theta_{i} z_{\bar{x} \widehat{x}, i}+\eta_{i} z_{\widehat{x}, i}+b_{i} z_{i}=-R_{i}, \quad i=\overline{1, N-1} \\
z_{0}=0, \\
z_{N}=\sum_{i=1}^{m-2} c_{i} z_{N_{i}},
\end{gathered}
$$

where $R_{i}$ is defined by 3.3 .

Lemma 4.1. The solution of the problem (4.1)-( $(4.3)$ satisfies the following estimates

holds.

$$
\|z\|_{\infty, \bar{\omega}_{N}} \leq C\|R\|_{\infty, \omega_{N}}
$$

Proof. According to the maximum principle, we have the following inequalities:

$$
\begin{gathered}
w(x)=z_{i}+\alpha^{-1}\|R\|_{\infty, \omega_{N}}, \\
w(0)=z_{0}+\alpha^{-1}\|R\|_{\infty, \omega_{N}} \geq 0,
\end{gathered}
$$

and

$$
w(1)=z_{N}+\alpha^{-1}\|R\|_{\infty, \omega_{N}} \geq 0,
$$

Next, from 4.5 and 4.7), we have

$$
L w(x)=z_{i}+\alpha^{-1}\|R\|_{\infty, \omega_{N}}=R_{i}+\alpha^{-1}\|R\|_{\infty, \omega_{N}} \geq 0
$$

and

which proves Lemma 4.1.

$$
\left\|z_{i}\right\| \leq \alpha^{-1}\|R\|_{\infty, \omega_{N}} \leq C\|R\|_{\infty, \omega_{N}}
$$

Lemma 4.2. Under the assumptions of Section 1 and Lemma 2.1, the solution of the problem (1.1)-(1.3) satisfies the following estimates for the remainder term $R_{i}$ :

$$
\left\|R_{i}\right\|_{L_{1}[0,1]} \leq C N^{-1} \ln N \text {. }
$$

We can state the convergence result of this study in the following Theorem.

Theorem 4.3. Let $u(x)$ be the solution of the problem (1.1)-(1.3) and $y_{i}$ be the solution of the difference scheme (3.6)-(3.8). Then, the following uniform error estimate satisfies

$$
\|y-u\|_{\infty, \bar{\omega}_{N}} \leq C N^{-1} \ln N
$$

\section{Algorithm and the Numerical Example}

In this part, an effective algorithm has been given for the solution of the difference scheme 3.6-3.8 and numerical results have also been displayed in table and graphs. 
5.1. Algorithm. We present the algorithm for the solution of the difference scheme (3.6)- 3.8 as

$$
\begin{gathered}
\left(\frac{\varepsilon \theta_{i}}{\hbar_{i} h_{i}}\right) y_{i-1}-\left(\frac{2 \varepsilon \theta_{i}}{h_{i} h_{i+1}}+b_{i}\right) y_{i}+\left(\frac{\varepsilon \theta_{i}}{\hbar_{i} h_{i+1}}\right) y_{i+1}=-f_{i}, \quad i=\overline{1, N-1} \\
y_{0}=0, y_{N}=d+y_{\frac{N}{4}}+2 y_{\frac{N}{3}}+3 y_{\frac{N}{2}}, \\
A_{i}=\frac{\varepsilon \theta_{i}}{\hbar_{i} h_{i}}, B_{i}=\frac{\varepsilon \theta_{i}}{\hbar_{i} h_{i+1}}, C_{i}=\frac{2 \varepsilon \theta_{i}}{h_{i} h_{i+1}}+b_{i} \\
\alpha_{i+1}=\frac{B_{i}}{C_{i}-A_{i} \alpha_{i}}, \beta_{i+1}=\frac{F_{i}+A_{i} \beta_{i}}{C_{i}-A_{i} \alpha_{i}}, \quad i=\overline{1, N-1} \\
y_{i}^{(n)}=\alpha_{i+1} y_{i+1}^{(n)}+\beta_{i+1}, \quad i=N-1, \ldots, 2,1 .
\end{gathered}
$$

5.2. Numerical Example. Here we examine the following test problem to see how the method works. We study the following test problem:

$$
\begin{gathered}
-\varepsilon u^{\prime \prime}(x)+u(x)=1, \quad 0<x<1 \\
u(0)=0, \quad u(1)=u\left(\frac{1}{2}\right)+2 u\left(\frac{1}{3}\right)+3 u\left(\frac{1}{2}\right)+d .
\end{gathered}
$$

We have the exact solution of this problem as:

$$
u(x)=\frac{\exp \left(-\frac{x}{\sqrt{\varepsilon}}\right)+\exp \left(\frac{x-1}{\sqrt{\varepsilon}}\right)}{1+\exp \left(-\frac{1}{\sqrt{\varepsilon}}\right)}-\cos ^{2}(\pi x) .
$$

The corresponding $\varepsilon$ uniform convergence rates are computed using the formula

$$
P^{N}=\frac{\ln \left(e^{N} / e^{2 N}\right)}{\ln 2} .
$$

The error estimates are denoted by

$$
e^{N}=\max _{\varepsilon} e_{\varepsilon}^{N}, \quad e_{\varepsilon}^{N}=\|y-u\|_{\infty, \bar{\omega}_{N}} .
$$

\section{Conclusion}

In this paper, we have offered a finite difference method for solving singularly perturbed convection-diffusion nonlocal boundary value problem. First, it is shown that the method displays uniform convergence with respect to the perturbation parameter $\varepsilon$. Then we have applied the present method on a test problem. In table and graphics, when $N$ takes increasing values, it is seen that the convergence rate of the smooth convergence speed $p^{N}$ is first order. The exact solution and approximate solution curves are almost identical as shown in Figure 1 . As $\varepsilon$ values decrease, the graph approaches more towards the coordinate axes in the boundary layer region around $x=1$. In Figure 2, the errors in these regions are maximum 
TABLE 1. The computed maximum pointwise errors $e^{N}$ and rates of convergence $p^{N}$

\begin{tabular}{cllllll}
\hline$\varepsilon$ & $N=24$ & $N=48$ & $N=96$ & $N=192$ & $N=384$ & $N=768$ \\
\hline $2^{-10}$ & 0.038865 & 0.019363 & 0.009294 & 0.004183 & 0.002034 & 0.001013 \\
& 1.00 & 1.05 & 1.15 & 1.04 & 1.00 & \\
$2^{-11}$ & 0.039397 & 0.019881 & 0.009801 & 0.004682 & 0.002103 & 0.001022 \\
& 0.98 & 1.02 & 1.06 & 1.15 & 1.04 & \\
$2^{-12}$ & 0.039664 & 0.020140 & 0.010055 & 0.004931 & 0.002349 & 0.001055 \\
& 0.97 & 1.00 & 1.02 & 1.06 & 1.15 & \\
$2^{-13}$ & 0.039797 & 0.020269 & 0.010181 & 0.005056 & 0.002474 & 0.001177 \\
& 0.97 & 0.99 & 1.00 & 1.03 & 1.07 & \\
$2^{-14}$ & 0.039863 & 0.020334 & 0.010244 & 0.005119 & 0.002535 & 0.001237 \\
& 0.97 & 0.98 & 1.00 & 1.01 & 1.03 & \\
$2^{-15}$ & 0.039897 & 0.020366 & 0.010274 & 0.005144 & 0.002566 & 0.001270 \\
& 0.97 & 0.98 & 0.99 & 1.00 & 1.01 & \\
$2^{-16}$ & 0.039914 & 0.020383 & 0.010302 & 0.005165 & 0.002576 & 0.001282 \\
& 0.96 & 0.98 & 0.99 & 1.00 & 1.00 & \\
$p^{N}$ & 0.96 & 0.98 & 0.99 & 1.00 & 1.00 & \\
\hline
\end{tabular}

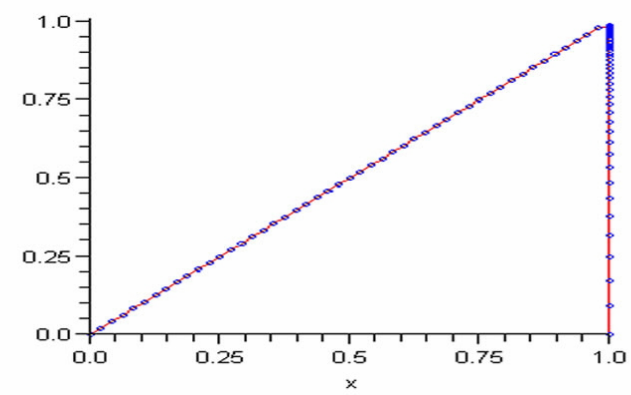

Figure 1. Comparison of approximate solution and exact solution for $\mathrm{N}=96, \varepsilon=2^{-16}$.

because of the irregularity caused by the sudden and rapid change of the solution in the boundary layer region around $x=1$ for different $\varepsilon$ values. Thus, numerical results show that the proposed scheme is working very well. All in all, we think that our study enhances academic understanding of the singularly perturbed problems with nonlocal condition. 


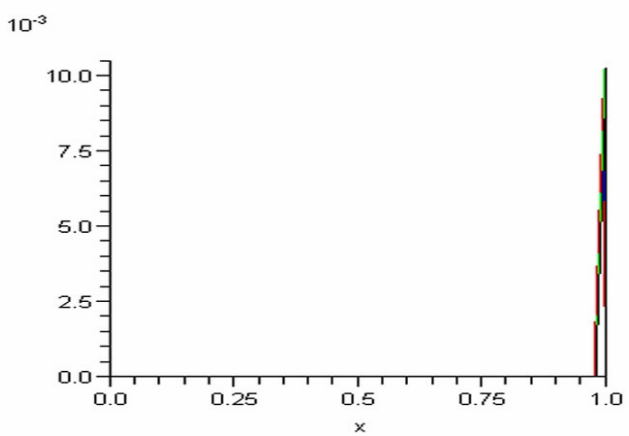

FiguRE 2. Error distribution for $\mathrm{N}=96, \varepsilon=2^{-10}, 2^{-12}, 2^{-14}, 2^{-16}$.

\section{REFERENCES}

[1] Adzic, N., Spectral approximation and nonlocal boundary value problems, Novi Sad J. Math. 30 (3), (2000), 1-10.

[2] Amiraliyev, G. M., Difference method for a singularly perturbed initial value problem, Turkish J. Math., 22, (1998), 283-294.

[3] Amiraliyev, G. M. and Cakir, M., A uniformly convergent difference scheme for singularly perturbed problem with convective term and zeroth order reduced equation, International Journal of Applied Mathematics, 2 (12), (2000), 1407-1419.

[4] Amiraliyev, G. M. and Cakir, M., Numerical solution of the singularly perturbed problem with nonlocal condition, Applied Mathematics and Mechanics (English Edition), 23 (7), (2002), 755-764.

[5] Arslan D., Finite difference method for solving singularly perturbed multi-point boundary value problem, Journal of the Institute of Natural and Applied Sciences, 22 (2), (2017), 64-75.

[6] Bakhvalov, N. S., On optimization of methods for solving boundary value problems in the presence of a boundary layer, Zhurnal Vychislitel'noi Matematikii Matematicheskoi Fiziki, 9 (4), (1969), 841-859.

[7] Bitsadze, A. V. and Samarskii, A. A., On some simpler generalization of linear elliptic boundary value problems, Doklady Akademii Nauk SSSR, 185, (1969), 739-40.

[8] Cakir, M. and Amiraliyev, G. M., Numerical solution of the singularly perturbed three-point boundary value problem, International Journal of Computer Mathematics, 84 (10), (2007), 1465-1481.

[9] Cakir, M., Uniform second-order difference method for a singularly perturbed three-point boundary value problem, Advances in Difference Equations, (2010), 13 pages.

[10] Cakir, M. and Amiraliyev, G. M., A numerical method for a singularly perturbed three-point boundary value problem, Journal of Applied Mathematics, (2010), 17 pages.

[11] Cakir, M. and Arslan, D. A numerical method for nonlinear singularly perturbed multi-point boundary value problem, Journal of Applied Mathematics and Phyics, 4, (2016), 1143-1156.

[12] Cakir, M. and Arslan, D. Numerical solution of the nonlocal singularly perturbed problem, Int. Journal of Modern Research in Engineering and Technology, 1, (5), (2016), 13-24.

[13] Cakir, M. and Arslan, D., Finite difference method for nonlocal singularly perturbed problem, Int. Journal of Modern Research in Engineering and Technology, 1, (5), (2016), 25-39. 
[14] Chegis, R., The numerical solution of problems with small parameter at higher derivatives and nonlocal conditions, Lietuvas Matematica Rinkinys, (in Russian), 28, (1988), 144-152.

[15] Farell, P. A., Miller, J. J. H., O'Riordan, E. and Shishkin, G. I., A uniformly convergent finite difference scheme for a singularly perturbed semilinear equation, SIAM Journal on Numerical Analysis, 33, (1996), 1135-1149.

[16] Gupta, C. P. and Trofimchuk, S. I., A sharper condition for the solvability of a three-point second order boundary value problem, Journal of Mathematical Analysis and Applications, 205, (1997), 586-597.

[17] Herceg, D. and Surla, K., Solving a nonlocal singularly perturbed nonlocal problem by splines in tension, Univ. u Novom Sadu Zb. Rad. Prirod.-Mat. Fak. Ser. Math., 21, (2), (1991), 119132.

[18] Miller, J. J. H., O'Riordan, E. and Shishkin, G. I., Fitted Numerical Methods for Singular Perturbation Problems, World Scientific, Singapore, 1996.

[19] Nayfeh, A. H., Introduction to Perturbation Techniques, Wiley, New York, 1993.

Current address: Derya Arslan (Corresponding Author): Department of Mathematics, Faculty of Art and Science, Bitlis Eren University, Bitlis, Turkey.

E-mail address: ayredlanu@gmail.com

ORCID Address: http://orcid.org/0000-0001-6138-0607

Current address: Musa Cakir: Department of Mathematics, Faculty of Science, University of Yuzuncu Yil, Van, Turkey,

E-mail address: cakirmusa@hotmail.com

ORCID Address: http://orcid.org/0000-0002-1979-570x 